\title{
Association Between 'Safer Sex Fatigue' and Rectal Gonorrhea Is Mediated by Unsafe Sex With Casual Partners Among HIV-Positive Homosexual Men
}

\author{
Stolte, Ineke G. MSc*; de Wit, John B. F. PhDt; Kolader, Marion MD‡; \\ Fennema, Han PhD‡; Coutinho, Roel A. Prof* $\$[/ /]$; Dukers, Nicole H. T. \\ M. PhD*§
}

From the *Cluster of Infectious Diseases, HIV \& STD Research, Municipal Health Service, Amsterdam, The Netherlands; the + Department of Social and Organizational Psychology, University of Utrecht, Utrecht, The Netherlands; the \# Cluster of Infectious Diseases, STD-Clinic, Municipal Health Service, Amsterdam, The Netherlands; the $\S$ Department of Human Retrovirology, Academic Medical Center, University of Amsterdam, Amsterdam, The Netherlands; and the [//] National Institute of Public Health and the Environment, Bilthoven, The Netherlands

The authors thank Titia Heijman and the other staff of the STD clinic for their contribution in data collection; the staff of the Public Health Laboratory of the Municipal Health Service for laboratory support; Ronald Geskus and Maria Prins for critically reading the manuscript; and Lucy Phillips for editing the final manuscript.

Grant no. 4014 from AIDS Fonds Netherlands has funded this research.

Correspondence: Ineke G. Stolte, MSc (Health Sciences), Municipal Health Service Amsterdam, Cluster of Infectious Diseases, HIV \& STD Research, Nieuwe Achtergracht 100, PO Box 22001000 CE Amsterdam, The Netherlands. E-mail: istolte@gggd.amsterdam.nl.

\section{ABSTRACT}

Objective: The objective of this study was to investigate whether and what sexual risk behavior is a mediator of associations between rectal gonorrhea (RG) and highly active antiretroviral therapy-related beliefs, safer sex fatigue, or sexual sensation-seeking among homosexual men.

Study Design: This study consisted of a cross-sectional survey between March 2002 and December 2003 among homosexual visitors of the Amsterdam sexually transmitted disease clinic.

Methods: In total, 1568 men answered a written questionnaire. Associations were determined using logistic regression corrected for repeated measurements.

Results: The RG infection rate was high among homosexual men who were HIVpositive $(16 \%)$ compared with those with negative or unknown HIV status. Mediation could be confirmed among HIV-positive men only. Those who experienced higher levels of safer sex fatigue were more likely to be positive for RG. This association was mediated by unprotected anal intercourse (UAI) with casual partners.

Conclusion: Addressing safer sex fatigue might help prevent UAI with casual partners and possibly also RG among HIV-positive homosexual men. 
Sex Transm Dis. 2006 Apr;33(4):201-8.

IN DEVELOPED COUNTRIES, THE availability of highly active antiretroviral therapy (HAART) has led to decreases in HIV/AIDS-related morbidity and mortality, 1 but has also coincided with increases in sexual risk behavior and sexually transmitted diseases (STDs) among homosexual men.2-8 Cross-sectional studies among these men have found associations between higher levels of sexual risk behavior and (perceived) viral load levels, motivational factors such as safer sex fatigue and sexual sensation-seeking, and also more optimistic beliefs about HAART.9-16 A meta-analytic review 17 recently confirmed higher levels of unprotected sex in persons who believed that receiving HAART or having an undetectable viral load reduced the risk of HIV transmission or whose concerns about engaging in unprotected sex were reduced by the availability of HAART. Causality, however, remained unclear in these studies. Results from a longitudinal study conducted in Amsterdam 18 indicated that perceiving less HIV/AIDS threat as a result of HAART availability might play a causal role in the increases in sexual risk behavior among HIV-negative homosexual men. Another study conducted in Amsterdam found that a more favorable perception of one's viral load level was associated with subsequent practice of unsafe sex among HIV-positive homosexual men.19

Whether and how increasing STD rates are associated with HAART-related beliefs, (perceived) viral load levels, and motivational factors has been studied less. One study conducted among initially HIV-negative homosexual men in Rotterdam found that perceiving less HIV/AIDS threat was associated with higher STD incidence and that perceiving less need for safer sex was associated with HIV seroconversion.20 The role of sexual risk behavior in the association between STD and HAART-related beliefs or motivational factors has not yet been investigated. It seems reasonable that the effect of HAART-related beliefs or motivational factors on STDs is partly explained or mediated by sexual risk behavior, which was not measured in the Rotterdam study. 20

Our study group studied homosexual men visiting the STD clinic in Amsterdam to investigate associations between an infection with rectal gonorrhea (RG) and HAART-related beliefs, safer sex fatigue, and sexual sensation-seeking. The main question was whether such an association is mediated by unprotected anal intercourse (UAI) with casual or steady partners. Additionally, this study aims to gain insight into how homosexual men differ regarding STD, HAART-related beliefs, safer sex fatigue, and sexual sensation-seeking according to their HIV status.

\section{Materials and Methods}

\section{Clinic Procedures}

The STD clinic in Amsterdam offers free STD diagnosis, treatment, and related services. Patients are routinely screened for (rectal) gonorrhea and infectious syphilis. At every new consultation, sociodemographic information is routinely collected and registered in a database under a patient identification code. An individual can have multiple new consultations in 1 year and/or more than one diagnosis per consultation. A positive culture is the diagnostic criterion for gonorrhea (GC-Lect agar, BBL; Becton Dickinson, Cockeysville, MD). 


\section{Study Procedures}

In the period between March 2002 and December 2003, 8166 new consultations were registered at the STD clinic in Amsterdam made by 5403 homosexual men. Homosexual men who could speak and write in Dutch were considered eligible for inclusion in current study and were randomly requested to fill in a questionnaire. This questionnaire included information about HIV status, sexual behavior, HAART-related beliefs, safer sex fatigue, and sexual sensation-seeking.

In total, 2991 questionnaires were distributed, of which 2544 could be matched to the STD registration system in the study period. The men who received the questionnaire differed from all homosexual men registered during the study period in being somewhat older (38.4 vs. 37.8, $P<0.01$ ), more often of Dutch nationality $(86.5 \%$ vs. $71.9 \%, P<0.01)$, and more often diagnosed with infectious syphilis $(5.9 \%$ vs. $4.6 \%, P=0.01)$ or RG $(7.2 \%$ vs. $5.7 \%, P=0.01)$.

\section{Study Population}

Of the 2544 questionnaires that were randomly distributed and could be matched to the STD registration system, 1568 (62\%) questionnaires were returned by 1391 homosexual men. Although some of these men (11.2\%) had more than one visit during the study period, all visits were considered as individuals.

Furthermore, 13 men were excluded as a result of a missing answer on the selfreported HIV status, yielding a convenience sample of 1555 homosexual men. These men were somewhat older $(39.1$ vs. $37.4, P<0.01)$ and more often of Dutch nationality $(89.9 \%$ vs. $81.0 \%, P<0.01)$ than men who received but did not return the questionnaire. The median age of the study participants was 38.7 (interquartile range [IQR], 32.8-44.4). Most men were of Dutch nationality, and the educational level was low (defined as lower vocational training or advanced elementary education) among $23 \%$, midlevel (defined as middle vocational training, higher general secondary education, secondary modern school, secondary grammar school) in $40.8 \%$ and high (defined as higher vocational training or university) among $36 \%$ of the men. A majority of the men were HIVnegative $(59.8 \%)$, whereas 222 men $(14.3 \%)$ were HIV-positive and 403 men (25.9\%) had an unknown HIV status.

\section{Dependent Variables}

\section{Rectal Gonorrhea.}

The infection rate of RG was defined as the total number of RG diagnoses in a given period per 100 consultations in that period.

\section{Sexual Risk Behavior.}

Participants were asked whether they had engaged in insertive or receptive anal intercourse in the previous 6 months and whether they had used condoms during anal intercourse. Risk behavior was defined as not consistently having used condoms during insertive or receptive anal intercourse; it was defined separately for steady and casual partners. Participants reporting no anal intercourse or consistent use of condoms during anal intercourse were considered not at risk. 
Sex Transm Dis. 2006 Apr;33(4):201-8.

\section{Predictor Variables.}

The questionnaire included items measuring HAART-related beliefs, to which participants responded using a seven-point scale ranging from $1=$ "strongly disagree" to 7 = "strongly agree."

Items were in part based on earlier studies 9,11,12 and were clustered based on principal component analyses, as was done in previous studies among homosexual men in Amsterdam.18,19 This approach confirmed four scales measuring different types of HAART-related beliefs: perceiving less HIV/AIDS threat since HAART (five items, Cronbach's [alpha] $=0.82$ ), perceiving less need for safer sex since HAART (three items, Cronbach's [alpha] $=0.88$ ), perceiving less risk for HIV transmission as a result of HAART (two items, correlation = 0.59), and perceiving high effectiveness of HAART in curing HIV/AIDS (three items, Cronbach's [alpha] $=0.84)$.

The questionnaire included measured the motivational factors of safer sex fatigue and sexual sensation-seeking as measured in other studies.11,21 Safer sex fatigue was measured using four items (e.g., "I feel tired of always having to monitor my sexual behavior") with a Cronbach's [alpha] of 0.87 , and sexual sensation-seeking was measured using two items (e.g., "I like wild and uninhibited sexual encounters") with a correlation of $r=0.84$.

The scores on all scales were calculated as the mean scores of the items included. A higher score represented a stronger agreement with the statements.

\section{Statistical Analyses}

Differences in sociodemographic characteristics, HAART-related beliefs, safer sex fatigue, sexual sensation-seeking, sexual risk behavior, and STD were examined according to HIV status using chi-squared tests for categorical variables and oneway analysis of variance (ANOVA) for continuous variables. Post hoc multiple comparisons analyses were conducted using Scheffé's test.

To investigate whether the possible association between HAART-related beliefs or motivational factors and RG infection is mediated by sexual risk behavior, the following assumptions must be confirmed. First, there has to be a univariate association between the predictor variable and RG infection showing an effect to be mediated. Second, there has to be a univariate association between the predictor variables and sexual risk behavior, the possible mediator. Third, sexual risk behavior (the possible mediator) has to be univariably associated with RG infection. Finally, the association between the predictor variable and RG infection has to decrease or disappear when sexual risk behavior is added to the model.22

The four assumptions were investigated using univariate and multivariate logistic regression. First, HAART-related beliefs, motivational factors, and sexual risk behavior were modeled as univariate predictors for RG infection (see Fig. 1A, C). Second, if a specific sexual behavior was found to be a risk factor for RG, HAARTrelated beliefs and motivational factors were each modeled as a predictor for that specific sexual risk behavior (see Fig. 1B). Third, mediation was tested in a multivariate model predicting RG. Only variables with a univariate $P$ value $<=0.10$ were included in the multivariate models, and they were built separately for HIVpositive men, HIV-negative men, and men with an unknown HIV status, because the three groups differ significantly. 
Sex Transm Dis. 2006 Apr;33(4):201-8.

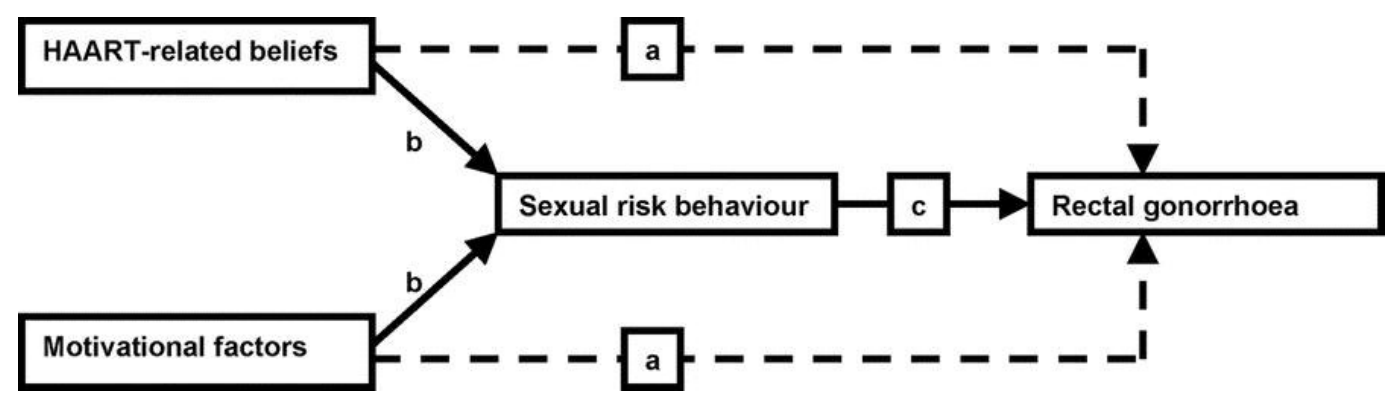

Fig. 1. (A-C) Mediation model

Because some of the men (11.3\%) had repeated visits during the study period, estimates and standard errors for all analyses were corrected for intraindividual correlation by using generalized estimating equations (GEE) assuming a compound symmetry correlation matrix.23 Analyses were done with the statistical packages SPSS 24 and Stata 7.0.23

\section{Results}

Sociodemographic and Behavioral Characteristics According to HIV Serostatus

Characteristics of all 1555 homosexual men are given in Table 1 according to their HIV status. HIV-positive men $(\mathrm{N}=222)$ were somewhat older and had a somewhat lower educational level than men who were HIV-negative $(\mathrm{N}=930)$ or had an unknown HIV status $(\mathrm{N}=403)$. Men with an unknown HIV status more often were of Dutch nationality than men with a positive or negative HIV status. 
Sex Transm Dis. 2006 Apr;33(4):201-8.

TABLE 1. Characteristics of 1555 Homosexual Visitors of the Amsterdam Sexually Transmitted Disease Clinic in the Period Between March 2002 and December 2003

\begin{tabular}{|c|c|c|c|c|c|c|}
\hline & $\begin{array}{l}\text { Total } \\
\text { Group }\end{array}$ & $\begin{array}{l}\text { HIV-Positive } \\
\text { Men }(\mathrm{N}=222)\end{array}$ & $\begin{array}{l}\text { HIV-Negative Men } \\
\quad(\mathrm{N}=930)\end{array}$ & $\begin{array}{l}\text { HIV-Unknown } \\
\text { Men }(\mathrm{N}=403)\end{array}$ & $\begin{array}{c}P \\
\text { Value }^{*}\end{array}$ & $\begin{array}{c}\text { Scheffé Post } \\
\text { Hoc Comparison }\end{array}$ \\
\hline \multicolumn{7}{|l|}{ Demographics } \\
\hline Age (median [IQR]) & $38.7(32.8-44.4)$ & $40.2(35.8-44.1)$ & $38.0(32.6-44.1)$ & $38.8(31.1-45.4)$ & $<0.01$ & $\mathrm{H}+>\mathrm{H}-, \mathrm{H} ?^{\dagger}$ \\
\hline Nationality (N [\%]) & & & & & $<0.01$ & \\
\hline Dutch & 1409 (89.9) & $198(89.2)$ & 820 (88.2) & $379(94.0)$ & & \\
\hline Non-Dutch & 159 (10.1) & $24(10.8)$ & $110(11.8)$ & $24(6.0)$ & & \\
\hline $\begin{array}{l}\text { Educational level" } \\
\qquad(\mathrm{N}[\%])\end{array}$ & & & & & $<0.01$ & \\
\hline Low & $355(23.1)$ & $76 / 220(34.5)$ & 191/909 (21.0) & 83/398 (20.9) & & \\
\hline Middle & $626(40.8)$ & $85 / 220(38.6)$ & $372 / 909$ (40.9) & $167 / 398(42.0)$ & & \\
\hline High & $553(36.0)$ & $59 / 220(26.8)$ & $346 / 909$ (38.1) & $148 / 398(37.2)$ & & \\
\hline \multicolumn{7}{|l|}{$\begin{array}{l}\text { HAART-related beliefs } \\
\text { (mean, SD) }\end{array}$} \\
\hline $\begin{array}{l}\text { Perceiving less HIV/ } \\
\text { AIDS threat since } \\
\text { HAART }\end{array}$ & $2.75(1.5)$ & $3.79(1.6)$ & $2.61(1.4)$ & $2.49(1.3)$ & $<0.01$ & $\mathrm{H}+>\mathrm{H}-, \mathrm{H}^{\dagger}$ \\
\hline $\begin{array}{l}\text { Perceiving less need } \\
\text { for safe sex since } \\
\text { HAART }\end{array}$ & $1.22(0.7)$ & $1.46(1.0)$ & $1.19(0.7)$ & $1.18(0.6)$ & $<0.01$ & $\mathrm{H}+>\mathrm{H}-, \mathrm{H}^{\dagger}$ \\
\hline $\begin{array}{l}\text { Perceiving less risk for } \\
\text { HIV transmission } \\
\text { as a result of } \\
\text { HAART }\end{array}$ & $2.45(1.5)$ & $2.45(1.5)$ & $2.42(1.5)$ & $2.51(1.5)$ & NS & \\
\hline $\begin{array}{l}\text { Perceiving high } \\
\text { effectiveness of } \\
\text { HAART in curing } \\
\text { HIV/AIDS }\end{array}$ & $1.65(1.1)$ & $1.51(1.0)$ & $1.66(1.1)$ & $1.71(1.1)$ & NS & \\
\hline \multicolumn{7}{|l|}{$\begin{array}{l}\text { Motivational beliefs } \\
\text { concerning sex } \\
\text { (mean, SD) }\end{array}$} \\
\hline Safer sex fatigue & $2.93(1.7)$ & $3.78(1.8)$ & $2.85(1.7)$ & $2.67(1.5)$ & $<0.01$ & $\mathrm{H}+>\mathrm{H}-, \mathrm{H}^{\dagger}{ }^{\dagger}$ \\
\hline $\begin{array}{l}\text { Sexual sensation- } \\
\text { seeking }\end{array}$ & $5.10(1.8)$ & $5.43(1.6)$ & $5.06(1.8)$ & $4.99(1.8)$ & 0.01 & $\mathrm{H}+>\mathrm{H}-, \mathrm{H}^{\dagger}$ \\
\hline \multicolumn{7}{|l|}{$\begin{array}{l}\text { Diagnoses of sexually } \\
\text { transmitted } \\
\text { diseases (N [\%]) }\end{array}$} \\
\hline Rectal gonorrhea & $115(7.3)$ & $35(15.8)$ & $52(5.6)$ & $28(6.9)$ & $<0.01$ & $\mathrm{H}+>\mathrm{H}-, \mathrm{H}^{\dagger}$ \\
\hline Infectious syphilis & $95(6.1)$ & $22(9.9)$ & $39(4.2)$ & $34(8.4)$ & $<0.01$ & $\mathrm{H}+, \mathrm{H}$ ? > $\mathrm{H}-\neq$ \\
\hline \multicolumn{7}{|l|}{$\begin{array}{l}\text { Sexual risk behavior } \\
\text { (N [\%]) }\end{array}$} \\
\hline $\begin{array}{l}\text { Unprotected anal } \\
\text { intercourse with } \\
\text { steady partners }\end{array}$ & $618 / 1546(40.0)$ & $92 / 218(42.1)$ & $391 / 915(42.7)$ & $131 / 400(32.8)$ & $<0.01$ & $\mathrm{H}+, \mathrm{H}->\mathrm{H} ?^{\S}$ \\
\hline $\begin{array}{l}\text { Unprotected anal } \\
\text { intercourse with } \\
\text { casual partners }\end{array}$ & 507/1509 (33.6) & $133 / 212(62.7)$ & $262 / 890(29.4)$ & 110/394 (27.9) & $<0.01$ & $\mathrm{H}+>\mathrm{H}-, \mathrm{H}^{\dagger}$ \\
\hline
\end{tabular}

${ }^{\star} P$ value is based on chi-squared tests for categorical variables and one-way analysis of variance for continuous variables.

${ }^{\dagger}$ HIV-positive men have a higher score than men with a negative or unknown HIV status.

${ }^{\ddagger} H I V$-positive men and men with an unknown HIV status have a higher score than HIV-negative men.

sHIV-positive and HIV-negative men have a higher score than men with an unknown HIV status.

Low = lower vocational training, or advanced elementary education; middle = middle vocational training, higher general secondary education, secondary modern school, secondary grammar school; high = higher vocational training or university.

IQR indicates interquartile range; HAART = highly active antiretroviral therapy; SD = standard deviation.

Homosexual men who were HIV-positive were more optimistic than men with a negative or unknown HIV status. They perceived less HIV/AIDS threat and less need for safer sex since HAART availability. They had a higher level of both safer sex fatigue and sexual sensation-seeking than did men with a negative or unknown HIV status.

Finally, HIV-positive men more often had UAI with casual partners than did men with a negative or unknown HIV status. UAI with steady partners, however, was reported most often by men with a positive or negative HIV status. The infection rate of infectious syphilis was highest among HIV-positive men (9.9\%) and among men with an unknown HIV status (8.4\%). The infection rate of RG was highest among HIV-positive men (15.8\%). 
Sex Transm Dis. 2006 Apr;33(4):201-8.

\section{Sexual Risk Behavior as a Mediator}

\section{HIV-Positive Homosexual Men.}

Univariate predictors for RG infection among HIV-positive homosexual men included no HAART use in the previous 6 months, reporting UAI with casual partners in the previous 6 months, and a higher level of safer sex fatigue (Table 2). In the multivariate model, no HAART use and UAI with casual partners were independent predictors for RG. The effect of safer sex fatigue decreased and became not statistically significant (Table 2 ) after inclusion of UAI with casual partners.

TABLE 2. Univariate and Multivariate Determinants for Rectal Gonorrhea and Unprotected Anal Intercourse With Casual Partners Among 222 HIV-Positive Homosexual Men Visiting the Amsterdam Sexually Transmitted Disease Clinic in the period March 2002 Through December 2003, The Netherlands

\begin{tabular}{|c|c|c|c|c|c|c|c|c|}
\hline & \multicolumn{4}{|c|}{$\begin{array}{c}\text { Rectal Gonorrhea } \\
\text { (infection rate: } 15.8 \% \text { ) }\end{array}$} & \multicolumn{4}{|c|}{$\begin{array}{l}\text { Unprotected Anal Intercourse } \\
\text { With Casual Partners }(62.7 \%)\end{array}$} \\
\hline & $\begin{array}{c}\text { OR } \\
(95 \% \mathrm{Cl})\end{array}$ & $\begin{array}{c}P \\
\text { Value }\end{array}$ & $\begin{array}{l}\text { OR adj } \\
(95 \% \mathrm{Cl})^{*}\end{array}$ & $\begin{array}{c}P \\
\text { Value }\end{array}$ & $\begin{array}{c}\text { OR } \\
(95 \% \mathrm{Cl})\end{array}$ & $\begin{array}{c}P \\
\text { Value }\end{array}$ & $\begin{array}{l}\text { OR adj } \\
(95 \% \mathrm{Cl})^{*}\end{array}$ & $\begin{array}{c}P \\
\text { Value }\end{array}$ \\
\hline \multicolumn{9}{|l|}{ Age } \\
\hline $\begin{array}{l}\text { Younger than } 40 \mathrm{y} \text {, older } \\
\text { than or equal } 40 \mathrm{y} \\
\text { Educational level }^{\dagger} \\
(\mathrm{N}[\%])\end{array}$ & $1.75(0.85-3.60) 1$ & 0.13 & & & & & & \\
\hline Low & 1 & 0.90 & & & $2.17(1.08-4.39)$ & $\neq$ & $3.23(1.45-7.17)$ & $\$$ \\
\hline Middle & $1.13(0.48-2.65)$ & & & & $1.83(0.90-3.71)$ & & $2.36(1.10-5.06)$ & $\neq$ \\
\hline $\begin{array}{l}\text { High } \\
\text { HAART-related beliefs } \\
\text { (mean, SD) }\end{array}$ & $1.22(0.52-2.86)$ & & & & 1 & 0.09 & & $<0.01$ \\
\hline $\begin{array}{l}\text { Perceiving less HIV/ } \\
\text { AIDS threat since } \\
\text { HAART }\end{array}$ & $1.16(0.90-1.49)$ & 0.26 & & & $1.38(1.15-1.66)$ & $<0.01$ & $1.08(0.88-1.32)$ & 0.46 \\
\hline $\begin{array}{l}\text { Perceiving less need } \\
\text { for safe sex since } \\
\text { HAART }\end{array}$ & $0.83(0.65-1.06)$ & 0.14 & & & $1.18(1.00-1.39)$ & 0.04 & $1.18(0.96-1.43)$ & 0.10 \\
\hline $\begin{array}{l}\text { Perceiving less risk for } \\
\text { HIV transmission as } \\
\text { a result of HAART }\end{array}$ & $1.25(0.95-1.64)$ & 0.11 & & & $1.83(1.06-3.15)$ & 0.03 & $1.23(0.75-2.00)$ & 0.43 \\
\hline $\begin{array}{l}\text { Perceiving high } \\
\text { effectiveness of } \\
\text { HAART in curing } \\
\text { HIV/AIDS }\end{array}$ & $1.08(0.83-1.40)$ & 0.57 & & & $1.00(0.76-1.31)$ & 0.98 & & \\
\hline \multicolumn{9}{|l|}{$\begin{array}{l}\text { Motivational beliefs } \\
\text { concerning sex } \\
\text { (mean, SD) }\end{array}$} \\
\hline Safer sex fatigue & $1.29(1.03-1.61)$ & 0.02 & $1.15(0.90-1.46)$ & 0.25 & $1.69(1.42-2.01)$ & $<0.01$ & $1.58(1.31-1.91)$ & $<0.01$ \\
\hline $\begin{array}{l}\text { Sexual sensation- } \\
\text { seeking }\end{array}$ & $1.08(0.83-1.40)$ & 0.57 & & & $1.17(1.00-1.37)$ & 0.05 & $1.16(0.96-1.39)$ & 0.12 \\
\hline \multicolumn{9}{|l|}{$\begin{array}{l}\text { Sexual risk behavior } \\
(\mathrm{N}[\%])\end{array}$} \\
\hline $\begin{array}{l}\text { No or protected anal } \\
\text { intercourse with } \\
\text { steady partners }\end{array}$ & 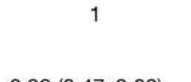 & 0.99 & & & & & & \\
\hline $\begin{array}{l}\text { Unprotected anal } \\
\text { intercourse with } \\
\text { steady partners }\end{array}$ & $0.99(0.47-2.08)$ & & & & & & & \\
\hline $\begin{array}{l}\text { No or protected anal } \\
\text { intercourse with } \\
\text { casual partners }\end{array}$ & 1 & 0.01 & 1 & $<0.01$ & & & & \\
\hline $\begin{array}{l}\text { Unprotected anal } \\
\text { intercourse with } \\
\text { casual partners }\end{array}$ & $3.24(1.29-8.15)$ & & $2.78(1.08-5.97)$ & & & & & \\
\hline \multicolumn{9}{|l|}{$\begin{array}{l}\text { Treatment with HAART } \\
\text { in the previous } 6 \\
\text { months }\end{array}$} \\
\hline $\begin{array}{l}\text { Yes } \\
\text { No }\end{array}$ & $2.61(1.28-5.31)$ & $<0.01$ & $\begin{array}{c}1 \\
2.78(1.08-5.97)\end{array}$ & 0.03 & $\begin{array}{c}1 \\
0.99(0.54-1.79)\end{array}$ & 0.96 & & \\
\hline
\end{tabular}

*Odds tatio adjusted for all variables with a univariate $P$ value $<0.10$.

'Low = lower vocational training or advanced elementary education; middle = middle vocational training, higher general secondary education, secondary modern school, secondary grammar school; high = higher vocational training or university.

${ }^{\ddagger} P$ value $<0.05$.

$\$ p$ value $<0.01$

$\mathrm{OR}$ indicates odds ratio; $\mathrm{OR}$ adj $=$ adjusted odds ratio; $95 \% \mathrm{Cl}=95 \%$ confidence interval; $\mathrm{HAART}=$ highly active antiretroviral therapy; $\mathrm{SD}=$ standard deviation.

Univariate risk factors for UAI with casual partners among HIV-positive homosexual men included: low educational level (vs. high educational level), perceiving less HIV/AIDS threat since availability of HAART, perceiving less risk 
for HIV transmission as a result of HAART, and perceiving less need for safer sex since HAART. Higher levels of safer sex fatigue and sexual sensation-seeking were likewise associated with UAI with casual partners (Table 2).

In multivariate analysis, only lower educational level and a higher level of safer sex fatigue were independently associated with more UAI with casual partners.

The association found between safer sex fatigue and RG infection in univariate analyses indicated that there was an effect that might have been mediated. This confirms the first assumption for mediation (see "Statistics") by which the other three assumptions that are needed to confirm mediation could be verified. Safer sex fatigue was associated with UAI with casual partners, the possible mediator, which in turn was associated with a higher infection rate of RG, confirming the second and third assumption for mediation. To confirm the fourth assumption, both UAI with casual partners and safer sex fatigue were included in a multivariate model predicting RG. The effect of safer sex fatigue on RG infection decreased and became nonsignificant, whereas UAI remained independently associated with RG. These results suggest that the association between safer sex fatigue and RG infection among HIV-positive homosexual men is indeed mediated by UAI with casual partners.

\section{HIV-Negative Homosexual Men.ㅍ}

Among HIV-negative homosexual men, univariate risk factors for RG infection included younger age, perceiving less risk for HIV transmission as a result of HAART, perceiving higher effectiveness of HAART in curing HIV/AIDS, and reporting UAI with steady partners in the previous 6 months (Table 3 ). In multivariate analysis, younger age and perceiving less risk for HIV transmission as a result of HAART were independent risk factors for RG. UAI with steady partners was marginally significantly associated with RG. 
Sex Transm Dis. 2006 Apr;33(4):201-8.

TABLE 3. Univariate and Multivariate Determinants of Rectal Gonorrhea (RG) Among 930 HIV-Negative and 403 HIV-Status-Unknown Homosexual Men Visiting the Sexually Transmitted Disease Clinic in Amsterdam in the Period Between March 2002 and December 2003, The Netherlands

\begin{tabular}{|c|c|c|c|c|c|c|c|c|}
\hline & \multicolumn{4}{|c|}{ HIV-Negative Homosexual Men } & \multicolumn{4}{|c|}{ Homosexual Men With an Unknown HIV Status } \\
\hline & \multicolumn{4}{|c|}{ Infection Rate RG: $5.6 \%$} & \multicolumn{4}{|c|}{ Infection Rate RG: $6.9 \%$} \\
\hline & $\begin{array}{c}\text { OR } \\
(95 \% \mathrm{Cl})\end{array}$ & $\begin{array}{c}P \\
\text { Value }\end{array}$ & $\begin{array}{l}\text { OR adj } \\
(95 \% \mathrm{Cl})^{*}\end{array}$ & $\begin{array}{c}P \\
\text { Value }\end{array}$ & $\begin{array}{c}\text { OR } \\
(95 \% \mathrm{Cl})\end{array}$ & $\begin{array}{c}P \\
\text { Value }\end{array}$ & $\begin{array}{l}\text { OR adj } \\
(95 \% \mathrm{Cl})^{*}\end{array}$ & $\begin{array}{c}P \\
\text { Value }\end{array}$ \\
\hline \multicolumn{9}{|l|}{ Age category } \\
\hline Less than $30 \mathrm{y}$ & $4.80(2.17-10.61)$ & $t$ & $4.26(1.89-9.64)$ & $\dagger$ & $6.12(1.90-19.64)$ & $\dagger$ & $5.28(1.65-16.97)$ & t \\
\hline $30-39$ y & $2.30(1.08-4.90)$ & $\neq$ & $2.30(1.06-4.99)$ & $\neq$ & $4.14(1.29-13.24)$ & $\neq$ & $3.60(1.11-11.66)$ & $\neq$ \\
\hline $\begin{array}{l}40 \text { y and older } \\
\text { HAART-related beliefs } \\
\text { (mean, SD) }\end{array}$ & 1 & $<0.01$ & 1 & $<0.01$ & 1 & $<0.01$ & 1 & 0.02 \\
\hline $\begin{array}{l}\text { Perceiving less HIV/ } \\
\text { AIDS threat since } \\
\text { HAART }\end{array}$ & $1.18(0.97-1.43)$ & 0.09 & $1.11(0.89-1.38)$ & 0.35 & $1.03(0.75-1.41)$ & 0.85 & & \\
\hline $\begin{array}{l}\text { Perceiving less need } \\
\text { for safe sex since } \\
\text { HAART }\end{array}$ & $1.27(0.95-1.69)$ & 0.11 & & & $0.68(0.21-2.19)$ & 0.51 & & \\
\hline $\begin{array}{l}\text { Perceiving less risk } \\
\text { for HIV } \\
\text { transmission as a } \\
\text { result of HAART }\end{array}$ & $1.28(1.09-1.51)$ & $<0.01$ & $1.22(1.01-1.49)$ & 0.04 & $1.09(0.86-1.38)$ & 0.48 & & \\
\hline $\begin{array}{l}\text { Perceiving high } \\
\text { effectiveness of } \\
\text { HAART in curing } \\
\text { HIV/AIDS }\end{array}$ & $1.21(1.00-1.47)$ & 0.05 & $1.11(0.87-1.42)$ & 0.40 & $0.91(0.64-1.29)$ & 0.60 & & \\
\hline \multicolumn{9}{|l|}{$\begin{array}{l}\text { Motivational beliefs } \\
\text { concerning sex } \\
\text { (mean, SD) }\end{array}$} \\
\hline Safer sex fatigue & $1.12(0.96-1.32)$ & 0.15 & & & $0.95(0.74-1.23)$ & 0.72 & & \\
\hline $\begin{array}{l}\text { Sexual sensation- } \\
\text { seeking }\end{array}$ & $1.06(0.89-1.26)$ & 0.50 & & & $1.01(0.82-1.25)$ & 0.60 & & \\
\hline \multicolumn{9}{|l|}{$\begin{array}{l}\text { Sexual risk behavior } \\
(\mathrm{N}[\%])\end{array}$} \\
\hline $\begin{array}{l}\text { No or protected anal } \\
\text { intercourse with } \\
\text { steady partners }\end{array}$ & 1 & 0.05 & 1 & 0.07 & 1 & 0.02 & 1 & 0.10 \\
\hline $\begin{array}{l}\text { Unprotected anal } \\
\text { intercourse with } \\
\text { steady partners }\end{array}$ & $1.78(1.00-3.17)$ & & $1.75(0.97-3.16)$ & & $2.54(1.17-5.51)$ & & $1.93(0.88-4.22)$ & \\
\hline $\begin{array}{l}\text { No or protected anal } \\
\text { intercourse with } \\
\text { casual partners }\end{array}$ & 1 & 0.90 & & & 1 & 0.57 & & \\
\hline $\begin{array}{l}\text { Unprotected anal } \\
\text { intercourse with } \\
\text { casual partners }\end{array}$ & $1.04(0.57-1.91)$ & & & & $0.76(0.30-1.95)$ & & & \\
\hline
\end{tabular}

${ }^{\star}$ Odds ratio adjusted for all variables with a univariate $P$ value $<0.10$.

${ }^{\dagger} P$ value $<0.01$

$\ddagger P$ value $<0.05$.

OR indicates odds ratio; $95 \% \mathrm{Cl}=95 \%$ confidence interval; $\mathrm{OR}$ adj = adjusted odds ratio; $\mathrm{HAART}=$ highly active antiretroviral therapy; $\mathrm{SD}=$ standard deviation.

Univariate risk factors for UAI with steady partners among HIV-negative homosexual men were younger age (odds ratio [OR], $1.73 ; 95 \%$ confidence interval $[\mathrm{CI}], 1.18-2.54)$, Dutch nationality (OR, 1.55; 95\% CI, 1.01-2.37), perceiving less HIV/AIDS threat (OR, $1.10 ; 95 \% \mathrm{CI}, 1.00-1.21)$, safer sex fatigue (OR, 1.18; 95\% CI, 1.09-1.28), and sexual sensation-seeking (OR, 1.08; 95\% $\mathrm{CI}, 1.00-1.16$ ). In multivariate analysis, only younger age (OR adjusted, 1.70; 95\% CI, 1.14-2.54) and safer sex fatigue (OR adjusted, $1.15 ; 95 \%$ CI, $1.06-$ 1.25 ) were independently associated with UAI with steady partners.

The univariate associations that were found between RG infection and, respectively, perceiving less risk for HIV transmission as a result of HAART and perceiving high effectiveness of HAART in curing HIV/AIDS pointed to effects that could have been mediated by UAI with steady partners (first assumption for mediation). However, because neither of the two beliefs was associated with UAI with steady partners (the possible mediator), mediation of the association between the two beliefs and RG could not be established among HIV-negative homosexual men in this study. 
Sex Transm Dis. 2006 Apr;33(4):201-8.

\section{Homosexual Men With an Unknown HIV Status.}

In homosexual men with an unknown HIV status, younger age and UAI with steady partners were associated with RG infection (Table 3). In multivariate analysis, only younger age was independently associated with RG.

Because none of the HAART-related beliefs or motivational factors was associated with RG infection, there was no effect that could have been mediated by sexual risk behavior among homosexual men with an unknown HIV status.

\section{Discussion}

The association between higher levels of sexual risk behavior and optimistic HAART-related beliefs, safer sex fatigue, and sexual sensation-seeking among homosexual men has been found by several studies.9-19 Because UAI is a predictor for STD, the findings of these studies often implied that factors like optimistic HAART-related beliefs contributed to increasing STD rates in industrialized countries. One study indeed found that perceiving less HIV/AIDS threat was associated with STD incidence, but it did not measure sexual risk behavior.20 To our knowledge, this cross-sectional study is the first to investigate associations between RG infection and HAART-related beliefs, safer sex fatigue, and sexual sensation-seeking while looking at sexual risk behavior among homosexual men according to their HIV status.

Results from the current study confirm that HIV-positive homosexual men are at higher risk for STD than those with a negative or unknown HIV status. The infection rates of RG and infectious syphilis were highest among HIV-positive men as were their levels of UAI, especially with casual partners, compared with men having a negative or unknown HIV status. Furthermore, HIV-positive men held more optimistic beliefs about HAART and reported higher levels of safer sex fatigue and sexual sensation-seeking. These findings are in accord with results from other studies comparing these three HIV-status groups.9-12,25

The risk of HIV transmission to uninfected casual partners could not be established in the current study, because the serostatus of casual partners was not collected. However, some HIV-positive homosexual men are known to engage in sexual behavior at risk for HIV transmission.26,27 Even if men want to avoid HIV transmission, they could hardly be sure of the HIV status of all casual partners, especially considering the relatively low HIV testing rates in Amsterdam.28 Because most HIV-positive men reporting UAI with casual partners engaged in both insertive and receptive UAI, ongoing HIV transmission is a substantial risk. Besides risking HIV transmission to others, HIV-positive men put themselves at risk for HIV treatment-resistant strains 29 and STD. Clearly, effective prevention programs must be developed that focus on the thoughts and needs of these men.

Behavioral risk factors for RG infection differed for homosexual men according to their HIV status. HIV-positive homosexual men having UAI with casual partners were at higher risk for RG than those not having UAI with casual partners. For HIV-negative men, UAI with steady partners was a risk factor for RG, which implies that these HIV-negative men or their steady partners probably also engage in UAI with other partners. Additional analysis among HIV-negative men indeed revealed that especially those reporting UAI with both steady and casual partners were at highest risk for RG infection (data not shown). Interestingly, HIV-positive men who were not on HAART in the previous 6 months were more likely to be infected with RG. 
Sex Transm Dis. 2006 Apr;33(4):201-8.

The main question, whether possible associations between RG infection and HAART-related beliefs and other motivational factors are mediated by sexual risk behavior, could be answered affirmatively among HIV-positive homosexual men. Our results suggest that the relation between safer sex fatigue and RG infection among these men is indeed mediated by UAI with casual partners. Possibly, HIVpositive homosexual men who experience a higher level of safer sex fatigue are more likely to engage in UAI with casual partners and, as a result, are at higher risk for infection with RG. However, such a causal relation cannot be proven in this study as a result of its cross-sectional nature. The few longitudinal studies investigating the association between beliefs and sexual risk behavior revealed inconclusive results.18-20,30 Huebner et al 30 suggest that treatment optimism may occur after sexual risk behavior, whereas authors of the other three studies suggest that treatment optimism precedes sexual risk behavior 18,19 or incident STD.20 Further longitudinal investigation is needed to increase our knowledge about the complex motivational processes underlying risky sexual behavior.

Although more optimistic beliefs about HAART were associated with UAI with casual partners among HIV-positive homosexual men, safer sex fatigue appears to be more important in understanding sexual risk behavior among these men. This was also found in another study among HIV-positive homosexual men 15 and is in line with the fact that among HIV-positive men in the current study, only safer sex fatigue was associated with RG infection, whereas none of the HAARTrelated beliefs were.

Among HIV-negative homosexual men, perceiving less risk for HIV transmission as a result of HAART was associated with a higher infection rate of RG. A recently conducted longitudinal study investigating the association between HAART-related beliefs and STD found that perceiving less HIV/AIDS threat was associated with incident STD among HIV-negative homosexual men in Rotterdam.20 This difference could be because the Rotterdam study included all STDs as an outcome, whereas our study specifically investigated RG as an outcome.

The relations between RG infection and optimistic beliefs in the current study, however, were not mediated by UAI with steady partners. It is nevertheless possible that another aspect of sexual risk behavior mediates the associations between the optimistic beliefs about HAART and RG such as UAI with (possibly) HIV-positive casual partners. Unfortunately, the current study did not collect information about UAI with (possibly) discordant casual partners, and mediation by such a specific sexual risk behavior could not be tested. Future research needs to investigate sexual risk behavior in more detail, because the various beliefs might be especially important when engaging in sexual intercourse at high risk for HIV transmission.

For men with an unknown HIV status, our results are limited. Those reporting UAI with steady partners were more likely to be infected with RG. However, multivariate analysis indicated that especially younger age is an important determinant for rectal gonorrhea. This is known to be an important determinant from other studies and was in our study also found for HIV-negative and HIVpositive homosexual men.

Obviously, care should be taken into generalizing the results of this study to all homosexual men in The Netherlands, let alone to those in other industrialized countries. First, homosexual visitors of the STD clinic may represent men who more often engage in risky sexual behavior and therefore are at higher risk for STDs than (Dutch) homosexual men in general. Second, men visiting the STD clinic who were included in the current study reported somewhat higher levels of 
Sex Transm Dis. 2006 Apr;33(4):201-8.

unsafe sex than did men who visited the STD clinic and were not included (see "Methods"). Furthermore, because HIV status was based on self-reported information, HIV-positive status might have been underreported. Questionnaires were anonymous, however, so response bias of HIV-positive status was likely to be reduced.

Despite the limitations of current study, results confirm the importance of developing interventions that address the needs of HIV-positive homosexual men in the era of HAART. Because the association between safer sex fatigue and RG is mediated by UAI with casual partners, addressing safer sex fatigue during counseling might help prevent UAI with casual partners among HIV-positive men and possibly also ongoing transmission of RG and other STDs. Furthermore, HIVpositive homosexual men engaging in high-risk sex need to become aware of their own risk for treatment-resistant strains and coinfection with STD, because these coinfections can have negative clinical consequences for HIV-positive individuals. Although the infection rate of RG and levels of UAI among men with a negative or unknown HIV status were lower than among HIV-positive men, prevention needs to keep addressing these men too. 
Sex Transm Dis. 2006 Apr;33(4):201-8.

\section{References}

1. Palella FJ, Delaney KM, Moorman AC, et al. Declining morbidity and mortality among patients with advanced human immunodeficiency virus infection. N Engl J Med 1998; 338:853-860.

2. Stolte IG, Dukers NHTM, de Wit JBF, et al. Increase in sexually transmitted infections among homosexual men in Amsterdam in relation to HAART. Sex Transm Infect 2001; 77:184-186.

3. Desquilbet L, Deveau C, Goujard C, et al. Increase in at-risk sexual behavior among HIV-1-infected patients followed in the French PRIMO cohort. AIDS 2002; 16:2329-2333.

4. Van de Ven P, Prestage G, Crawford J, et al. Sexual risk behavior increases and is associated with HIV optimism among HIV-negative and HIV-positive gay men in Sydney over the 4-year period to February 2000. AIDS 2000; 14:2951-2953.

5. Centers for Disease Control and Prevention. Trends in primary and secondary syphilis and HIV infections in men who have sex with men-San Francisco and Los Angeles, California, 1998-2002. MMWR Morb Mortal Wkly Rep 2004; 53:575-578.

6. Elford J, Bolding G, Davis M, et al. Trends in sexual behavior among London homosexual men 1998-2003: Implications for HIV prevention and sexual health promotion. Sex Transm Infect 2004; $80: 451-454$.

7. Bij AK, Stolte IG, Dukers NHTM, Coutinho RA. Increase of sexually transmitted infections, but not HIV, among young homosexual men in Amsterdam: Are STIs still reliable markers for HIV transmission? Sex Transm Infect 2005; 81:34-37.

8. Dodds JP, Nardone A, Mercey DE, Johnson AM. Increase in high risk sexual behavior among homosexual men, London 1996-8: Cross sectional, questionnaire study. BMJ 2000; 320:1510-1511.

9. Vanable PA, Ostrow DG, McKirnan DJ, et al. Impact of combination therapies on HIV risk perceptions and sexual risk among HIV-positive and HIV-negative gay and bisexual men. Health Psychol 2000; 19:134-145.

10. Elford J, Bolding G, Maguire M, Sherr L. Combination therapies for HIV and sexual risk behavior among gay men. J Acquir Immune Defic Syndr 2000; 23:266-271.

11. Ostrow DE, Fox KJ, Chmiel JS, et al. Attitudes towards highly active antiretroviral therapy are associated with sexual risk taking among HIV-infected and -uninfected homosexual men. AIDS 2002; $16: 775-780$.

12. Van de Ven P, Kippax S, Knox S, et al. HIV treatments optimism and sexual behavior among gay men in Sydney and Melbourne. AIDS 1999; 13:2289-2294.

13. Kalichman SC, Nachimson D, Cherry C, Williams E. AIDS treatment advances and behavioral prevention setbacks: Preliminary assessment of reduced perceived threat of HIV-AIDS. Health Psycho $1998 ; 17: 546-550$.

14. Vanable PA, Ostrow DG, McKirnan DJ. Viral load and HIV treatment attitudes as correlates of sexual risk behavior among HIV-positive gay men. J Psychosom Res 2003; 54:263-269.

15. Cox J, Beauchemin J, Allard R. HIV status of sexual partners is more important than antiretroviral treatment related perceptions for risk taking by HIV positive MSM in Montreal, Canada. Sex Transm Infect 2004; 80:518-523.

16. Miller M, Meyer $L$, Boufassa $F$, et al. Sexual behavior changes and protease inhibitor therapy. AIDS 2000; 14:F33-39.

17. Crepax N, Hart TA, Marks G. Highly active antiretroviral therapy and sexual risk behavior, a metaanalytic review. JAMA 2004; 292:224-236. 
Sex Transm Dis. 2006 Apr;33(4):201-8.

18. Stolte IG, Dukers NHTM, Geskus RB, et al. Homosexual men change to risky sex when perceiving less threat of HIV/AIDS since availability of highly active antiretroviral therapy: A longitudinal study. AIDS 2004; 18:303-309.

19. Stolte IG, de Wit JBF, van Eeden A, et al. Perceived viral load, but not actual HIV-1-RNA load, is associated with sexual risk behavior among HIV-infected homosexual men. AIDS 2004; 18:19431949.

20. Van der Snoek EM, de Wit JBF, Mulder PGH, van der Meijden WI. Incidence of sexually transmitted disease and HIV infection related to perceived HIV/AIDS threat since highly active antiretroviral therapy availability in men who have sex with men. Sex Transm Dis 2005; 32:170-175.

21. DiFranceisco W, Ostrow DG, Chmiel JS. Sexual adventurism, high-risk behavior, and human immunodeficiency virus-1 seroconversion among the Chicago MACS-CCS cohort, 1984-1992. A casecontrol study. Sex Transm Dis 1996; 23:453-460.

22. Kenny DA, Kashy DA, Bolger N. Data analysis in social psychology. In: Gilbert D, Fiske S, Lindzey G, eds. The Handbook of Social Psychology, vol 1, 4th ed. Boston: McGraw-Hill, 1998:233-265.

23. Stata Statistical Software: Release 7.0. College Station, TX, State Corp.

24. SPSS Inc. SPSS for windows, release 9.0.0.

25. Kim AA, Kent CK, Klausner JD. Risk factors for rectal gonococcal infection amidst resurgence in HIV transmission. Sex Transm Dis 2003; 30:813-817.

26. Parsons JT, Schrimshaw EW, Wolitski RJ, et al. Sexual harm reduction practices of HIVseropositive gay and bisexual men: Serosorting, strategic positioning, and withdrawal before ejaculation. AIDS 2005; 19(suppl 1):S13-25.

27. Bachmann LH, Grimley DM, Waithaka $Y$, et al. Sexually transmitted disease/HIV transmission risk behaviors and sexually transmitted disease prevalence among HIV-positive men receiving continuing care. Sex Transm Dis 2005; 32:20-26.

28. Hospers HJ, Dörfler TT, Zuilhof W. Monitor Research Amsterdam 2004 Factsheets (in Dutch) Schorer, Unigraph Maastricht. Available at:

http://www.monitoronderzoekonline.nl/downloads/monitor-ams2004-factsheets.pdf . Accessed May 2005.

29. Little SJ, Holte S, Routy J, et al. Antiretroviral-drug resistance among patients recently infected with HIV. N Engl J Med 2002; 347:385-394.

30. Huebner DM, Rebchook GM, Kegeles SM. A longitudinal study of the association between treatment optimism and sexual risk behavior in young adult gay and bisexual men. J Acquir Immune Defic Syndr 2004; 37:1514-1519. 